\title{
PRODUÇÃO DE BIODIESEL 3G POR PHORMIDIUM AUTUMNALE A PARTIR DE RESÍDUOS AGROINDUSTRIAIS
}

\author{
C. NEVES ${ }^{1}$, M. M. MARONEZE ${ }^{1}$, G. J. G. TEIXEIRA, L. Q. ZEPKA ${ }^{1}$ e E. JACOB-LOPES ${ }^{1}$ \\ ${ }^{1}$ Universidade Federal de Santa Maria, Departamento de Tecnologia e Ciência de Alimentos \\ E-mail para contato: jacoblopes@pq.cnpq.br
}

\begin{abstract}
RESUMO - O objetivo do trabalho foi avaliar a produção de biodiesel 3G partir de efluentes do abate e processamento de bovinos por Phormidium autumnale. Os experimentos foram realizados em biorreatores de coluna de bolhas, nas condições de $100 \mathrm{mg} / \mathrm{L}$ de inóculo, $\mathrm{pH}$ ajustado a 7,5, reator isotérmico operando a $20^{\circ} \mathrm{C}$, aeração contínua de 1VVM e ausência de luminosidade. Os resultados demonstraram uma produtividade em biomassa de $29,7 \mathrm{mg} / \mathrm{L} . \mathrm{d}$ em paralelo a uma produtividade lipídica de $4,57 \mathrm{mg} / \mathrm{L} . \mathrm{d}$. Os ácidos graxos de maior representatividade foram os ácidos oléico, palmitoléico e esteárico com $27 \%, 15 \%$ e $13 \%$, respectivamente. As propriedades de combustão do biodiesel $3 \mathrm{G}$ indicaram adequacidade em relação às normas internacionais para biodiesel, demostrando o potencial de exploração desta rota tecnológica.
\end{abstract}

\section{INTRODUÇÃO}

Os resíduos agroindustriais são resultado de diversas operações unitárias do processamento industrial. A indústria de processamento de carne utiliza aproximadamente 62 milhões de $\mathrm{m}^{3}$ por ano de água no mundo. No entanto, apenas uma pequena quantidade tornase componente do produto final, e a fração restante converte-se em águas residuárias com concentrações elevadas de material em suspensão, elevada carga de matéria orgânica e rica também em nitrogênio e fósforo (Sroka et al., 2004). O lançamento destes efluentes em corpos hídricos proporciona alterações na concentração de oxigênio dissolvido, além de possibilitar a eutrofização de corpos hídricos (Demirbas, 2011).

As microalgas possuem substancial capacidade de bioconversão de material orgânico e nutrientes presentes em águas residuárias. Neste sentido, sua utilização em efluentes agroindustriais seria uma alternativa em relação às formas convencionais de tratamento, uma vez que possuem menores custos e são mais eficientes. Estes sistemas permitem a remoção de matéria orgânica e nitrogênio em uma única etapa, produzindo uma biomassa com valor econômico devido ao seu teor significativo de proteínas e lipídeos (Queiroz et al., 2007; Markou \& Georgakakis, 2011).

A grande dependência pelos combustíveis fósseis levou ao surgimento de discussões sobre questões como a poluição atmosférica e a escassez dos recursos energéticos, direcionando ao desenvolvimento de tecnologias para a obtenção de fontes de energias alternativas e renováveis, como os biocombustíveis de $1^{\text {a }}$ a $4^{\text {a }}$ geração (Martín \& Grossmann, 2012). As microalgas têm sido apontadas como uma fonte renovável capaz de atender grande 
parte da demanda global de combustíveis para transportes, uma vez que são caracterizados pelas elevadas taxas de crescimento e elevado teor de óleo intracelular (Chisti, 2007).

Em face disso, o objetivo do estudo foi avaliar a produção de biodiesel de $3^{\mathrm{a}}$ geração produzido a partir do cultivo heterotrófico da microalga Phormidium autumnale, empregando resíduos agroindustriais como fonte de carbono orgânico.

\section{MATERIAL E MÉTODOS}

\subsection{Microorganismo, meio de cultivo e água residuária}

A microalga utilizada foi a Phormidium autumnale obtida a partir de um isolamento de uma área localizada no deserto de Cuarto Cienégas $\left(26^{\circ} 59^{\prime} \mathrm{N}, 102^{\circ} 03^{\prime} \mathrm{W}\right.$ - México). $\mathrm{O}$ meio de cultura utilizado foi o meio sintético BGN (Rippka et al., 1979) adicionado de agaragar na concentração de $15 \mathrm{~g} / \mathrm{L}$.

O efluente proveniente do abate e processamento de bovinos foi coletado após o tratamento primário (peneira rotativa e equalizador), na estação de tratamento de efluentes de uma indústria localizada na cidade de Santa Maria, RS.

\subsection{Biorreator}

O aparato experimental foi constituído de um biorreator do tipo coluna de bolhas, construído em vidro de $0,5 \mathrm{~mm}$ de espessura, diâmetro interno de $15 \mathrm{~cm}$, altura de $20 \mathrm{~cm}$ e 3,5 $\mathrm{L}$ de volume de trabalho. O sistema de dispersão de gases do reator constituiu em um difusor de ar de 1,5 cm localizado no centro da base da coluna.

\subsection{Dados cinéticos, Métodos Analíticos e Parâmetros Cinéticos}

Os experimentos foram conduzidos em um biorreator de coluna de bolhas construído em vidro de $0,5 \mathrm{~mm}$ de espessura, diâmetro interno de $6,5 \mathrm{~cm}$, altura de $70 \mathrm{~cm}$ e $2,5 \mathrm{~L}$ de volume/ de trabalho. As condições experimentais utilizadas foram: concentração inicial do inóculo de $100 \mathrm{mg} / \mathrm{L}$, pH de 7,6, aeração constante de 1VVM (volume de ar por volume de meio por minuto) e ausência de luminosidade.

As amostras foram coletadas em intervalos regulares de 12 horas e caracterizadas quanto a demanda química de oxigênio (DQO), determinados conforme a metodologia descrita no Standard Methods for the Examination of Water and Wastewater (APHA, 2005). A concentração celular foi determinada gravimetricamente por meio da filtração de um volume conhecido de efluente em filtro de $0,45 \mu \mathrm{m}$ de diâmetro. A composição centesimal foi feita baseada na metodologia descrita na A.O.A.C (2000). Os testes foram realizados em duplicata e os dados cinéticos referentes à média de quatro repetições.

A fração lipídica da biomassa foi extraída pelo método de Bligh \& Dyer (1959). O método de Hartman e Lago (1976) foi utilizado para saponificar e esterificar o extrato seco de lipídeos para obtermos os ésteres metílicos de ácidos graxos (biodiesel). A composição de ácidos graxos foi determinada usando o cromatógrafo a gás VARIAN 3400CX (Varian, Palo Alto-CA, USA). Os ésteres metílicos dos ácidos graxos foram identificados por comparação 
dos tempos de retenção com os tempos padrão (Supelco, Louis-MO, USA) e quantificados por normalização de área. Adicionalmente, as propriedades de combustão do biodiesel foram analisadas BiodieselAnalizer ${ }^{\odot}$ proposto por Biofuel Research Team (BRTeam), que analisou os parâmetros de combustão do biodiesel.

\section{RESULTADOS E DISCUSSÃO}

Os parâmetros cinéticos para o cultivo de Phormidium autumnale, a partir de resíduos do abate e processamento de bovinos estão apresentados na Tabela 1.

Tabela 1 - Parâmetros cinéticos do processo

\begin{tabular}{cc}
\hline Parâmetros & Valor \\
\hline $\mathrm{r}_{\mathrm{S}(\mathrm{DQO})}(\mathrm{mg} / \mathrm{L} . \mathrm{d})$ & 68,5 \\
$\mathrm{P}_{\mathrm{X}}(\mathrm{mg} / \mathrm{L} . \mathrm{d})$ & 29,7 \\
Lipídeos $(\%)$ & 15,4 \\
$\mathrm{P}_{\mathrm{L}}(\mathrm{mg} / \mathrm{L} . \mathrm{d})$ & 4,57 \\
\hline
\end{tabular}

O cultivo de Phormidium autumnale em águas residuárias do processamento de abate de bovinos apresentou taxa de consumo do substrato de $68,5 \mathrm{mg} / \mathrm{L} . \mathrm{d}$, produtividade em biomassa de $29,7 \mathrm{mg} / \mathrm{L}$.d, teor de lipídeos de $15,4 \%$ e produtividade lipídica na ordem de 4,57 $\mathrm{mg} / \mathrm{L} . d$. Estudos indicam conteúdos lipídicos provenientes de células das microalgas variáveis, em média, de 20\% a 40\% em termos de biomassa seca (Ma e Hanna, 1999; Luque et al., 2010; Mairet et al., 2011). Esses microorganismos podem produzir de 25 a 220 vezes mais triacilglicerídeos do que plantas oleaginosas terrestres (Ahmad et al., 2011).

A qualidade dos óleos unicelulares é um fator decisivo na escolha e definição de rotas tecnológicas de produção de biodiesel $3 \mathrm{G}$, independente dos aspectos quantitativos. Neste sentido, a Tabela 2, apresenta o perfil de ácidos graxos da microalga cultivada a partir de resíduos agroindustriais.

Tabela 2 - Perfil de ácidos graxos da Phormidium autumnale a partir de resíduos agroindustriais

\begin{tabular}{cc}
\hline Ácidos Graxos & Percentual (\%) \\
\hline $\mathrm{C} 16: 1$ & 15,2 \\
$\mathrm{C} 17: 0$ & 8,8 \\
$\mathrm{C} 18: 0$ & 13,5 \\
$\mathrm{C} 18: 1 \mathrm{n} 9 \mathrm{c}$ & 27,3 \\
$\mathrm{C} 18: 2 \mathrm{n} 6 \mathrm{c}$ & 5,3 \\
\hline Saturados & 22,30 \\
Monoinsaturados & 42,50 \\
Poliinsaturados & 5,30 \\
\hline
\end{tabular}


O perfil de ácidos graxos do óleo microalgal é o principal fator que determina as características do biodiesel. Ao total, identificaram-se três diferentes ácidos graxos majoritários: ácido oléico $(27,3 \%)$, ácido palmitoléico $(15,2 \%)$ e ácido esteárico $(13,5 \%)$. Dessa forma, o perfil de ácidos graxos foi predominantemente saturado $(22,30 \%)$ e monoinsaturado (42,50\%). Knothe (2005) reporta que óleos com composição predominantemente saturada e monoinsaturada são os mais adequados para a síntese de biodiesel, pois produzem combustíveis com propriedades ideais ao uso em motores a diesel.

Adicionalmente, a Tabela 3 apresenta as propriedades de combustão do biodiesel obtido a partir da microalga Phormidum autumnale, comparado ao biodiesel de soja.

Tabela 3 - Propriedades de combustão do biodiesel obtido pela microalga Phormidium autumnale, a partir resíduos agroindustriais

\begin{tabular}{cccccc}
\hline $\begin{array}{c}\text { Propriedades do } \\
\text { biodiesel }\end{array}$ & $\begin{array}{c}\text { Biodiesel } \\
\mathbf{3 G}\end{array}$ & $\begin{array}{c}\text { Biodiesel } \\
\text { de soja }^{\mathbf{1}}\end{array}$ & ANP 255 & $\begin{array}{c}\text { ASTM } \\
\mathbf{6 7 5 1}\end{array}$ & EN 14214 \\
\hline $\mathrm{CE}(\%)$ & 99,8 & 96,6 & - & - & $\min .96,5$ \\
$\mathrm{NC}$ & 55,0 & 49,0 & $\min .45$ & $\min .47$ & $\operatorname{min.~} 51$ \\
$\mathrm{II}\left(\mathrm{gI}_{2} 100 \mathrm{~g}^{-1}\right)$ & 49,33 & 128,0 & - & - & $\max .120$ \\
$\mathrm{GI}(\%)$ & 53,1 & 143,8 & - & - & - \\
$\mathrm{IS}$ & 142,96 & & - & - & - \\
$\mathrm{FCC}(\%)$ & 6,75 & & - & - & - \\
$\mathrm{PEFF}\left({ }^{\circ} \mathrm{C}\right)$ & 4,73 & $-5,0$ & $\operatorname{max.} 2$ & - & - \\
$\mathrm{PN}\left({ }^{\circ} \mathrm{C}\right)$ & $-4,99$ & & & - & - \\
$\mathrm{PAE}$ & 37,9 & & - & - & - \\
$\mathrm{PBAE}$ & 5,3 & & - & - & - \\
$\mathrm{EO}(\mathrm{h})$ & 0 & & - & - & - \\
$\mathrm{VA}$ & 27,69 & & - & - & - \\
$\mu\left(\mathrm{mm}^{2} / \mathrm{s}\right)$ & 0,97 & & 1 & - & - \\
$\rho\left(\mathrm{g} / \mathrm{cm}^{3}\right)$ & 0,61 & & - & - & - \\
\hline
\end{tabular}

CE: conteúdo de ésteres, NC: número de cetano, II: índice de iodo, GI: grau de instauração, IS: índice de saponificação, FCC: fator de comprimento da cadeia, PEFF: ponto de entupimento de filtro a frio, PN: ponto de névoa, PAE: posição alílica equivalente, PBAE: posições bis-alílicas equivalente, EO: estabilidade oxidativa, VA: valor de aquecimento, $\mu$ : viscosidade cinemática e $\rho$ : densidade.

${ }^{1}$ Ramos et al., (2009).

Verifica-se que o biodiesel produzido a partir da biomassa da Phormidium autumnale gerada durante o tratamento de resíduos do abate e processamento de bovinos possui um conteúdo de ésteres de 99,9\%, um número de cetano de 55,0, um índice de iodo de $49,33 \mathrm{gI}_{2} / 100 \mathrm{~g}^{-1}$, um grau de insaturação de $53,1 \%$, índice de saponificação de 142,96 , fator de comprimento de cadeia de $6,75 \%$, ponto de entupimento de filtro a frio de $-5,0^{\circ} \mathrm{C}$, ponto de névoa de $-4,99^{\circ} \mathrm{C}$, posição alílica equivalente de 37,9 , posições bis-alílicas equivalentes de 5,3 , estabilidade oxidativa de 0 horas, valor de aquecimento de 27,69, viscosidade cinemática de $0,97 \mathrm{~mm}^{2} / \mathrm{s}$ e densidade de $0,61 \mathrm{~g} / \mathrm{cm}^{3}$. 
Os valores das propriedades de combustão do biodiesel obtidos no presente estudo são superiores aos exigidos pela norma brasileira (ANP 255), norte americana e europeia (ASTM 6751 e EN 14214), respectivamente. Esses resultados sugerem a potencialidade de exploração do biodiesel obtido a partir da biomassa da Phormidium autumnale nas condições avaliadas.

\section{CONCLUSÃO}

A síntese e caracterização do biodiesel microalgal, evidenciou a possibilidade da obtenção de um combustível com características de qualidade (teor de ésteres de 99,9\%, número de cetano de 55,0, índice de iodo de $49,33 \mathrm{gI}_{2} / 100 \mathrm{~g}^{-1}$, grau de insaturação de $53,1 \%$, ponto de entupimento de filtro a frio de $-2,8^{\circ} \mathrm{C}$, ponto de névoa de $-4,99^{\circ} \mathrm{C}$, posição alílica equivalente de 37,9 , posições bis-alílicas equivalentes de 5,3 , estabilidade oxidativa de 0 horas, valor de aquecimento de 27,69 , viscosidade cinemática de $0,97 \mathrm{~mm}^{2} / \mathrm{s}$ e densidade de $0,61 \mathrm{~g} / \mathrm{cm}^{3}$ ) enquadradas dentro das normas brasileiras, europeias e norte americanas, possibilitando a reutilização da biomassa gerada no processo de tratamento na direção da síntese de biocombustíveis como produto final do processo global de tratamento.

\section{REFERENCIAS}

AHMAD, A.L.; YASIN, N.H.M.; DEREK, C.J.C.; LIM, J.K. Microalgae as a sustainable energy source for biodiesel production: a review. Renewable and Sustainable Energy Reviews, v. 15, p. 584-593, 2011.

AMERICAN PUBLIC HEALTH ASSOCIATION- APHA. Standard Methods for the Examination of Water and Wastewater. 20. ed. Washington: APHA, 2005.

ANP (Agência Nacional de Petróleo, Gás Natural e Biocombustíveis). Boletim mensal do biodiesel. 2013.

ASSOCIATION OF OFFICIAL ANALYTICAL CHEMISTS. Official methods of analysis of the Association of Official Analytical Chemists. 17. ed. Gaithersburg, Maryland: AOAC, 2000.

ASTM 6751. Standard Specification for Biodiesel Fuel (B100). Blend Stock for Distillate Fuels, 2002.

BLIGH, EG; DYER, JW. A rapid method of total lipid extraction and purification. Journal Biochem Phisiol 37:911-917, 1959.

BOHDZIEWICZ, J.; SROKA, E. Application of hybrid systems to the treatment of meat industry wastewater. Desalination v.198, p.33-40, 2006.

CHISTI, Y. Biodiesel from microalgae. Biotechnology Advances, v.25, p.294-306, 2007.

HARTMAN, L.; LAGO, R.C.A. A rapid preparation of fatty acid methyl esters from lipids. Laboratory Pratice, v.22, p.475-476, 1976. 
KNOTHE, G. Dependence of biodiesel fuel properties on the structure of fatty acid alkyl esters. Fuel Processing Technology, v. 86, p.1059-1070, 2005.

LUQUE, R.; LOVETT, J.C.; DATTA, B.; CLANCY, J.; CAMPELO, J.M.; ROMERO, A.A. Biodiesel as feasible petrol fuel replacement: a multidisciplinary overview. Energy Environmental Science, v. 3, p. 1706-1721, 2010.

MA, F.; HANNA, M.A. Biodiesel production: a review. Bioresource Technolony, v. 70, p. 1$15,1999$.

MAIRET, F.; BERNARD, O.; MASCI, P.; LACOUR, T.; SCIANDRA, A. Modelling neutral lipid production by the microalga Isochrysis aff. galbana under nitrogen limitation. Bioresource Technology, v. 102, p. 142-149, 2011.

MARKOU, G.; GEORGAKAKIS, D. Cultivation of filamentous cyanobacteria (blue-green algae) in agro-industrial wastes and wastewaters: A review. Applied Energy, v.88, p.3389-3401, 2011.

MARTÍN, M.; GROSSMANN, I.E. On the synthesis of sustainable biorefi neries. Industrial \& Engineering Chemistry Research, v.52, p.3044-3064, 2012.

QUEIROZ, M.I.; JACOB-LOPES, E.; ZEPKA, L.Q.; BASTOS, R.G.; GOLDBECK, R. The kinetics of the removal of nitrogen and organic matter from parboiled rice effluent by cyanobacteria in a stirred batch reactor. Bioresource Technology v.98, p.2163-2169, 2007.

RAMOS, M. J.; FERNANDEZ, C. M.; CASAS, A.; RODRIGUEZ, R.; PEREZ, A. Influence of fatty acid composition of raw materials in biodiesel properties. Bioresource Technology, n.100, p.261-268, 2009.

RIPPKA, R. et al. Generic Assignments Strain Histories and Properties of Pure Cultures of Cyanobacteria. Journal of General Microbiolog. Great Britain. n.111. p.1-61, 1979.

SROKA, A.; KAMINSKI,W.; BOHDZIEWICZ, J. Biological treatment of meat industry wastewater. Desalination, v.162, p.85-91, 2004.

UNE-EN 14214. Automotive Fuels, Fatty Acid Methyl Esters (FAME) for Diesel Engines, Requirements and Test Methods, 2003. 